Françoise Pecker Aissata Amadou Sandrine Magne Catherine Pavoine

\section{ADRESSES}

F. Pecker: directeur de recherche à l'Inserm. A. Amadou: étudiante en thèse. S. Magne: étudiante en thèse. $\mathrm{C}$. Pavoine: chargée de recherche à l'Inserm. Inserm U. 99, IM3, Hôpital HenriMondor, 51, avenue du Maréchal-de-Lattrede-Tassigny, 94010 Créteil, France.

TIRÉS À PART

F. Pecker.

$m / s n^{\circ} 10$, vol. 14 , octobre 98

\title{
Le rôle messager de l'acide arachidonique dans le cardiomyocyte
}

L'acide arachidonique est un constituant structural majeur des membranes et un précurseur essentiel de nombreuses molécules bioactives (eicosanoïdes). C'est aussi un second messager intracellulaire. Sous l'effet des phospholipases A2 (PLA2), C ou D, l'acide arachidonique est libéré des phospholipides membranaires en réponse à plusieurs hormones et dans certaines conditions pathologiques, l'ischémie en particulier. Son rôle de second messager dans les cardiomyocytes est à l'ordre du jour: il pourrait être le médiateur de l'apoptose déclenchée par le TNF $\alpha$, comme cela a été décrit dans les cellules leucémiques. Produit sous l'action de la PLA2, il active la sphingomyélinase qui hydrolyse la sphingomyéline et libère le céramide. L'AA est aussi le second messager du mini-glucagon, fragment carboxy-terminal du glucagon: le mini-glucagon stimule la PLA2 et entraîne une libération graduée de l'AA membranaire ; l'AA, comme le mini-glucagon, agit en synergie avec le glucagon et l'AMP cyclique pour augmenter l'amplitude des oscillations calciques et la contraction des cellules stimulées électriquement (action inotrope positive). Les cibles de l'AA pourraient être la protéine-kinase $\mathrm{C}$ et les canaux ioniques.

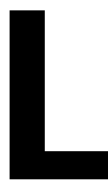

'acide arachidonique (AA, acide gras polyinsaturé C20, 4 doubles-liaisons: 5, 8, 11, 14) est présent en grande quantité dans le sérum, complexé à l'albumine. Lorsqu'il est capté par la cellule, il est essentiellement stocké dans les phospholipides membranaires sous forme estérifiée (résidu arachidonyl). La phospholipase-A2 cytosolique (cPLA2), qui hydrolyse préférentiellement les liaisons sn2-arachidonyl des glycérophospholipides (voir figure 1), joue un rôle central dans le relargage d'AA provoqué par les facteurs de croissance ou les neuromédiateurs. Son implication dans des maladies aussi diverses que l'allergie, l'ischémie cérébrale, l'inflammation, la diminution de fécondité ou le retard de parturition, vient d'être confirmée par les études faites sur des souris transgéniques, déficientes en cPLA2 [1, 2]. Les études biochimiques ont par ailleurs montré que l'activité cPLA2 est augmentée, dans le tissu cérébral, au cours de différentes atteintes neu- 
rodégénératives et, dans le tissu cardiaque, au cours d'épisodes d'ischémie/reperfusion. L'AA est utilisé par la cellule comme source d'énergie, via la $\beta$-oxydation mitochondriale, ou comme précurseur des eicosanoïdes (prostaglandines leucotriènes, époxydes...). Ces deux fonctions ont longtemps occulté les effets de l'AA lui-même sur des systèmes enzymatiques et des canaux ioniques : ils ont été facilement attribués à des métabolites ou considérés comme des « effets lipides», ou " effets membranaires » pouvant être reproduits par d'autres lipides. Mais plusieurs résultats récents décrivent des effets propres de l'AA. Des cibles de l'AA ont été identifiées : la protéine-kinase C dans différents tissus, NFKB, la sphingomyélinase dans des cellules leucémiques humaines, des canaux ioniques de cellules neuronales, de cardiomyocytes, de myocytes du muscle lisse, de cellules pulmonaires, la phosphatase de la chaîne légère de la myosine du muscle lisse. Enfin, l'AA relaie la réponse apoptotique au tumor necrosis factor (TNF $\alpha$ ) de cellules leucémiques humaines et module la transmission glutamatergique. Ce constituant membranaire, cet intermédiaire métabolique, peutil être élevé au rang "noble » de messager? Cette revue se propose de faire le point sur le rôle messager de l'AA dans le cardiomyocyte.

\section{Les seconds messagers de la signalisation hormonale}

Trois types de voies de signalisation couplées à des récepteurs membranaires ont été décrits et existent dans le cardiomyocyte. (1) Le premier type s'appuie sur des molécules «seconds messagers", nucléotides ou ions. Le patriarche de ces seconds messagers est l'AMP cyclique, messager des récepteurs à sept segments membranaires, couplés à la protéine Gs et à l'adénylyl cyclase (par exemple, les récepteurs $\beta$-adrénergiques). L'AMP cyclique a pour cible la protéine-kinase A dont les substrats principaux, dans la cellule cardiaque, sont les canaux calciques L de la membrane du sarcolemme, le phospholamban (inhibiteur de la pompe à $\mathrm{Ca}^{2+}$ du réti-

I, la phosphorylase-kinase, la glycogène synthase et la lipase [3]. Le GMP cyclique est le messager de l'ANF (atrial natriuretic factor) et du NO qui activent la guanylyl cyclase. Dans le cardiomyocyte, le GMP cyclique a trois cibles principales: les phosphodiestérases de types 2 et 3 , respectivement activées et inhibées par le nucléotide [4] et la protéinekinase $\mathrm{G}[5]$. Les ions $\left(\mathrm{Ca}^{2+}, \mathrm{K}^{+}, \mathrm{Na}^{+}\right)$ jouent aussi des rôles de seconds messagers, en réponse à l'activation et à l'ouverture de canaux [6]. (2) La deuxième voie de transmission du signal n'emprunte pas de molécules «messagers» mais implique des cascades de phosphorylation mises en route par l'activation de récepteurs qui ne traversent qu'une fois la membrane. Certains ont une activité kinase intrinsèque, tyrosine-kinase (récepteur du type de celui de l'insuline [7]) ou sérine/thréonine kinase (récepteurs de cytokines de la famille du TGF- $\beta$ [8]). D'autres recrutent une protéine-kinase (récepteurs des cytokines de la famille des interleukines [9]). (3) Enfin, une troisième voie de transmission du signal met en jeu des messagers lipidiques. Ces messagers incluent l'inositol trisphosphate (IP3), le diacylglycérol (DAG) [10], l'acide lysophosphatidique (LPA), les eicosanoïdes [11], le céramide [12]. Leur particularité est qu'ils ne sont pas confinés au milieu intracellulaire, comme les nucléotides ou les ions, mais peuvent passer la membrane cellulaire et jouer des rôles de messagers intra- et intercellulaires, tel l'IP3, ou intra- et extracellulaires, telles les prostaglandines. La fonction de messager, telle qu'elle a été définie par Sutherland et reprise dans une revue récente de Khan $e t$ al. (Durham, NC, USA) [11] répond à quatre requis: (1) des stimulus physiologiques modulent la concentration cellulaire du messager; (2) le messager ou des analogues reproduisent les effets physiologiques; (3) des cibles cellulaires interagissent directement avec le messager; (4) enfin, ces cibles transmettent une réponse cellulaire. En ce qui concerne les messagers lipidiques, le premier requis est, généralement, le premier à être vérifié. Mais les requis (2) et (3) sont plus difficiles à prouver du fait de la fonction d'intermédiaires métaboliques de ces lipides. Le rôle messager de l'IP3, dans la mobilisation $\mathrm{du} \mathrm{Ca}^{2+}$ intracellulaire et la communication intercellulaire, a été établi grâce à l'identification de récepteurs propres qui ont été caractérisés et clonés. Les effets du diacylglycérol (DAG), qui active la protéine-kinase $\mathrm{C}$, et ceux du céramide, médiateur de l'apoptose, sont reproduits par des molécules synthétiques, perméantes: les esters de phorbol pour le premier, le céramide C2 ou C6 pour le second. En revanche, le seul analogue de l'AA, disponible aujourd'hui, est l'ETYA (C20; 4 triples liaisons: 5, 8, 11, 14). Cependant, il n'active pas la PKC, cible potentielle de l'AA.

\section{L'homéostasie de I'AA dans le cardiomyocyte}

La capacité du cardiomyocyte de synthétiser des acides gras de novo est faible. L'AA sérique, du fait de son caractère lipophile, passe librement la membrane cellulaire. Mais, dans le cardiomyocyte et dans des conditions physiologiques normales, l'AA est essentiellement incorporé sous forme estérifiée dans les phospholipides membranaires ; l'AA non estérifié représente moins de $0,1 \%$ de l'AA cellulaire total. L'arachidonyl constitue environ $30 \%$ des chaînes hydrocarbonées des glycérophospholipides et est retrouvé principalement en position sn-2. La majorité de l'AA libéré en réponse à un stimulus résulte donc de l'activation de phospholipases (figure 1): libération directe par les PLA2 qui hydrolysent spécifiquement les liaisons sn-2; libération indirecte par les phospholipases-C qui libèrent du DAG à partir duquel l'AA peut être produit par action des DAG- ou MAG-lipases; libération indirecte aussi par les phospholipases-D qui produisent de l'acide phosphatidique susceptible d'être converti en DAG. La concentration d'AA libre dépend par ailleurs de sa réincorporation dans les phospholipides (réacylation), de sa liaison à des protéines de liaison des acides gras (FABP) et de son métabolisme par quatre voies possibles d'oxydation: cyclo-oxygénation, lipoxygénation, mono-oxygénation par les cytochromes P-450 et $\beta$-oxydation mitochondriale.

Les effets hormonaux relayés par l'AA peuvent être couplés à l'activa- 


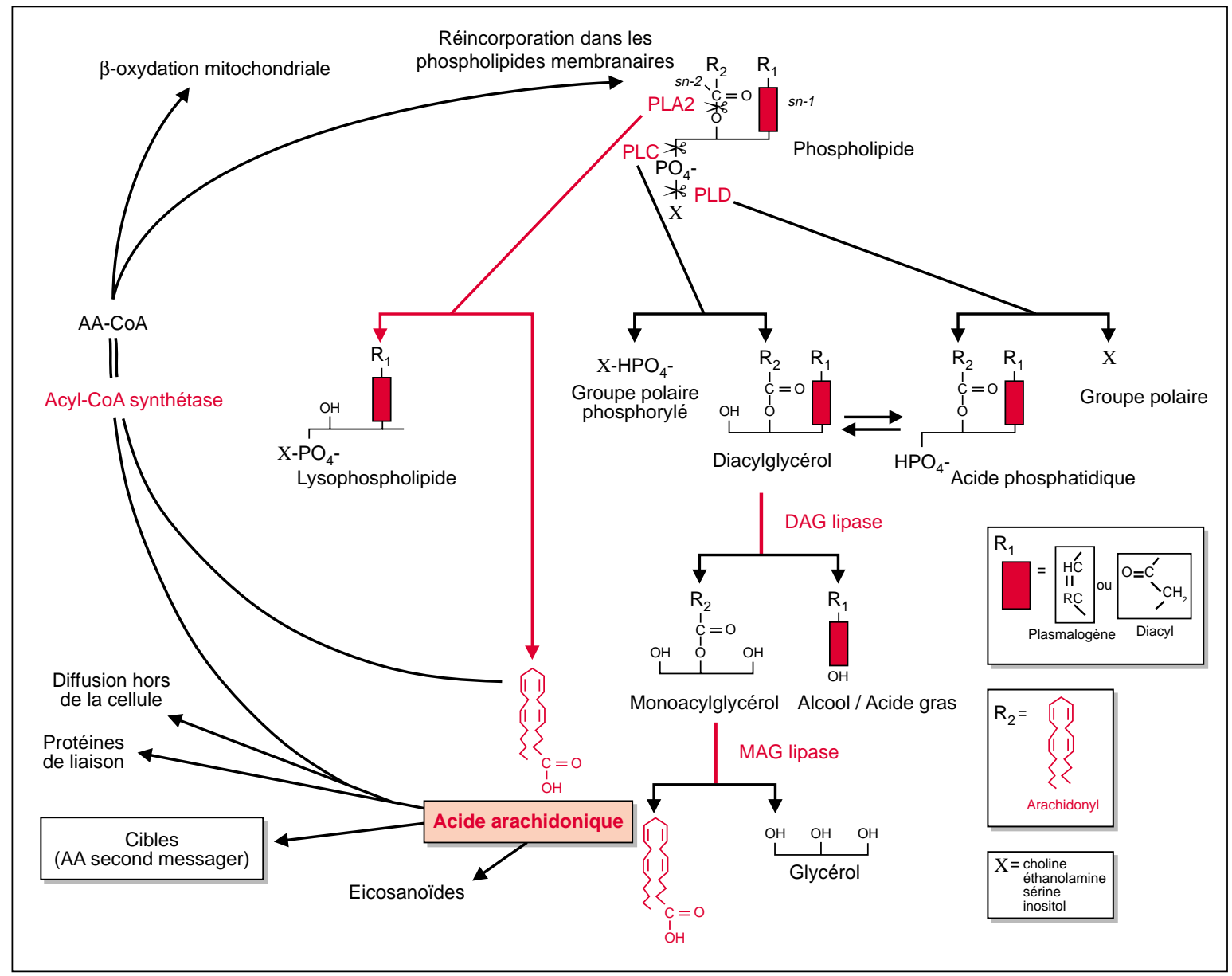

Figure 1. Les voies de relargage de l'acide arachidonique (AA). La source majeure de I'AA cellulaire est l'AA sérique. Il est stocké sous forme estérifiée dans les phospholipides membranaires, le résidu arachidonyl représentant environ $30 \%$ des chaînes carbohydrates en position sn-2 des glycérophospholipides. La production d'AA par les phospholipases-A2 (PLA2) est directe. Mais il peut être produit de façon indirecte par l'action des phospholipases C et D (PLC, PLD). DAG : diacylglycérol, MAG, monoacylglycérol.

tion des phospholipases $\mathrm{C}$ et $\mathrm{D}$, mais sont surtout liés à l'activation de PLA2. Les PLA2 se répartissent en deux grandes classes : les PLA2 sécrétées (sPLA2) et les PLA2 cellulaires (cPLA2 et iPLA2 [13-15]). Les sPLA2 sont des enzymes de petit poids moléculaire (13-18 kDa), extracellulaires, retrouvées dans les venins de serpent, le pancréas de mammifère, le liquide synovial. Elles sont très impliquées dans les réponses inflammatoires et ne présentent pas de spécificité vis-à-vis du groupe acyl en position sn-2 des phospholipides; leur activité est maximale en présence de concentrations millimolaires de Ca. Les PLA2 cellulaires ont des poids moléculaires plus élevés que les PLA2 sécrétées. La PLA2 cyto- solique (cPLA2), dont le poids moléculaire est de $85 \mathrm{kDa}$ d'après l'ADN complémentaire mais qui, dans beaucoup d'exemples, migre sur gel SDS comme une protéine de 100-110 kDa [13], est ubiquiste. Elle présente une très forte sélectivité pour les phospholipides contenant une chaîne arachidonyl en position sn-2. Elle est susceptible de plusieurs types de régulations, sur lesquelles nous reviendrons et qui l'impliquent dans un certain nombre d'actions hormonales $[16,17]$. Une autre PLA2 cellulaire (iPLA2), identifiée dans le tissu cardiaque [18], a un poids moléculaire de $40 \mathrm{kDa}$, mais existe sous forme de complexe, associée à la phospho-fructokinase. Elle est stimulée par l'ATP, est sélective pour les dérivés plasmalogènes contenant des résidus arachidonyl et est augmentée lors d'épisodes ischémiques. Son activité ne nécessite pas l'ajout de $\mathrm{Ca}^{2+}$ : cette propriété la distingue de la cPLA2 et lui a valu son appellation de PLA2 indépendante du $\mathrm{Ca}^{2+}$, iPLA2. Cependant, le groupe de Gross (Saint-Louis, MO, USA) vient de montrer l'association, dépendante du $\mathrm{Ca}^{2+}$, de cette enzyme avec la calmoduline [18]. L'activité enzymatique est stimulée lorsque le complexe se dissocie, en présence d'inhibiteurs de calmoduline [18] ou lors d'une libération de $\mathrm{Ca}^{2+}$ des réservoirs intracellulaires [19]; cette dernière observation, faite dans les cellules musculaires lisses, est récente et est la seule qui lie l'activation de la 
iPLA2 de 40 kDa à des altérations de l'homéostasie calcique. Enfin, une PLA2 cytosolique, également cataloguée comme iPLA2, vient d'être clonée: elle a une masse moléculaire de $85 \mathrm{kDa}$ mais forme des oligomères de 270-350 kDa, possède huit motifs ankyrine, n'a pas d'homologie de séquence avec les autres phospholipases et est exprimée dans tous les tissus. Elle présente une spécificité vis-à-vis de l'acide phosphatidique 1,2-dipalmitoyl et produirait donc préférentiellement de l'acide lysophosphatidique [20].

Jusqu'à présent, la cPLA2 a été le point de mire des études d'action hormonale attachées à l'AA. En effet, l'enzyme présente une spécificité visà-vis des phospholipides contenant de l'AA en position sn-2; elle est, en outre, la cible de plusieurs types de régulations (voir [13]): (1) en présence de concentrations $\mu \mathrm{M}$ de $\mathrm{Ca}^{2+}$, la cPLA2 subit une translocation du cytosol vers son substrat, dans les membranes cellulaires; (2) elle est activée par phosphorylation et est un substrat des MAP-kinases; (3) la protéine-kinase $\mathrm{C}$ jouerait aussi un rôle dans l'activation de la cPLA2 mais, plutôt qu'une activation directe, la protéine-kinase $\mathrm{C}$ déclencherait une cascade de phosphorylations conduisant à l'activation des MAP-kinases; (4) le couplage de la cPLA2 à des protéines G a même été décrit [21]. Cependant, il n'a pas été prouvé que ce couplage était direct. De fait, dans d'autres tissus que le coeur, l'activation de récepteurs couplés aux protéines $\mathrm{Gi} / \mathrm{Gq}$, qui relaient la stimulation de la phospholipase C $\beta$ et la mobilisation de $\mathrm{Ca}^{2+}$ intracellulaire, produit une libération d'AA, et cet effet est reproduit, dans les cellules CHO, par le ionophore calcique A23187 [22]. Dans un esprit de simplification il est tentant, comme Clark et al. (Cambridge, MA, USA) [16] le proposent, d'introduire les protéines $\mathrm{G}$ en amont d'une cascade phospholipase-C - protéine-kinase C - MAPkinase - cPLA2 ou d'une voie MAPkinase-cPLA2 (figure 2); (5) enfin, à côté des voies de stimulation directes, post-transcriptionnelles, l'expression de la cPLA2 est contrôlée par des cytokines et des facteurs de croissance (voir [16]).

Des publications des années 1977- la bradykinine, l'endothéline 1 produisent une accumulation d'AA dans le cour perfusé. Mais seuls quelques résultats récents concernent la stimulation hormonale de l'activité PLA2 propre des cardiomyocytes. Ainsi, l'angiotensine II [23-25], la bradykinine [26] et l'étirement [27] stimulent l'activité PLA2 des cardiomyocytes. Dans ces études, cependant, le type de PLA2 impliqué n'a pas été identifié. En revanche, il a été montré que le TNF $\alpha$ et les agonistes $\alpha 1$-adrénergiques augmentaient l'expression de la sPLA2 dans les cardiomyocytes de rat nouveau-né [28]. La thrombine [29], l'interleukine-1 $\beta$ [30] et l'ischémie $[29,31]$ stimulent l'activité de la iPLA2 de $40 \mathrm{kDa}$. Le mini-glucagon active la cPLA2 par un mécanisme qui implique une protéine G [25].

Ces résultats mettent en avant le rôle cible des PLA2 et les variations de concentration cellulaire d'AA libre qu'entraînent certains stimulus hormonaux ou pathologiques. Ils introduisent le rôle potentiel de second messager de l'AA.

\section{L'AA médiateur de l'apoptose}

L'AA joue un rôle de médiateur dans l'apoptose. Le groupe d'Hannun
(Durham, NC, USA) a d'abord démontré l'implication du céramide dans la réponse apoptotique au TNF $\alpha$ des cellules HL-60 (m/s 1993, $n^{\circ} 6-7, p$. 813) [32]. Le céramide est un produit de l'hydrolyse de la sphingomyéline par la sphingomyélinase. Son rôle d'intermédiaire, non seulement dans l'apoptose mais aussi dans les réponses à différents stress $(\mathrm{m} / \mathrm{s}$ 1996, $n^{\circ}$ 5, p. 658) [12], a été établi sur les quatre critères que doit remplir un second messager: (1) l'apoptose est précédée par une accumulation de céramide; cela a été observé dans plusieurs types cellulaires et en réponse à différents stimulus; (2) les analogues perméants du céramide (tels le céramide C2 ou C6) induisent l'apoptose; (3) des systèmes enzymatiques, cibles du céramide ont été identifiés qui incluent une sérinethréonine phosphatase, la ceramideactivated protein phosphatase (CAPP) [12] ; (4) il y a une réponse cellulaire à la stimulation par les analogues du céramide : l'apoptose. Cependant des études récentes du même groupe ont montré que l'activation de la sphingomyélinase par le TNF $\alpha$ était relayée par l'AA [33] et impliquait l'activation de la cPLA2 [34]. Il est intéressant de noter que la cPLA2 est aussi le système effecteur dans

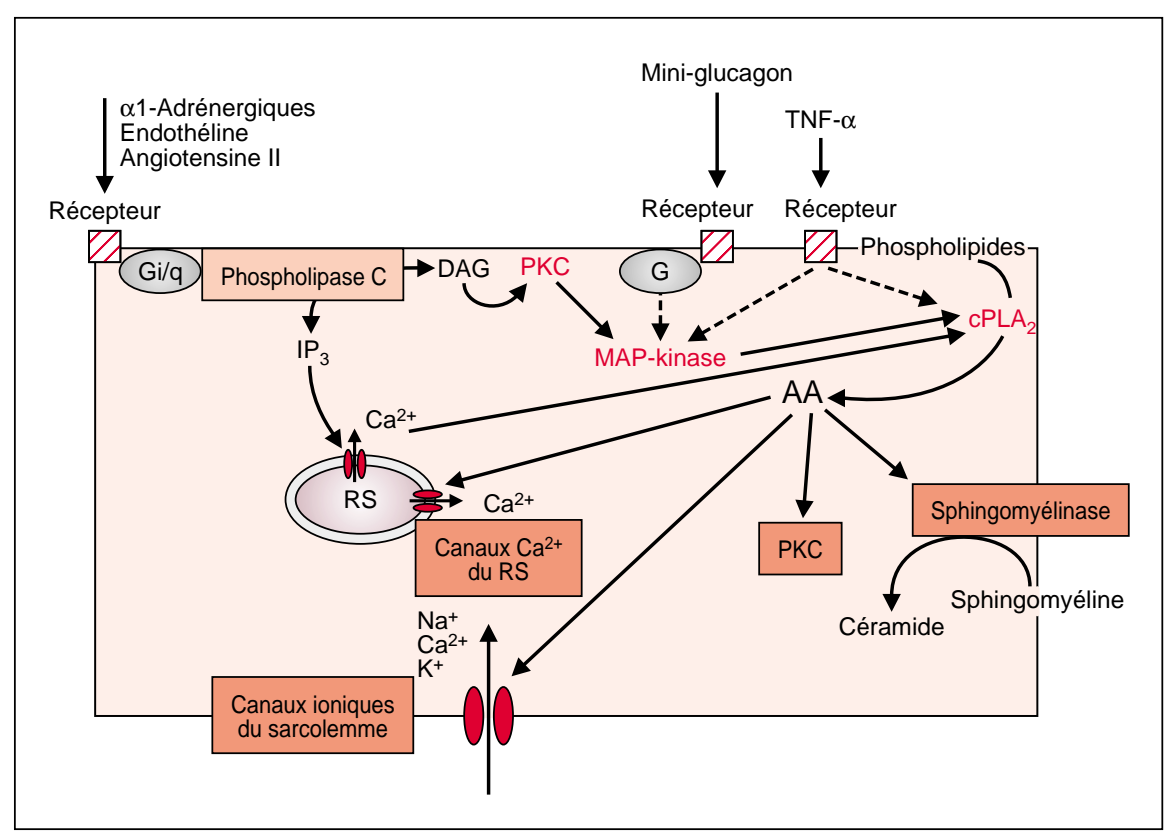

Figure 2. Les cibles potentielles de I'acide arachidonique (AA). Voies d'activations hormonales proposées pour le relargage de I'AA via la phospholipase-A2 cytosolique (cPLA2) et cibles potentielles de I'AA. PKC: protéinekinase $C ; R S$ : réticulum sarcoplasmique. 
l'action cytotoxique du TNFo [35]. Dans la cascade des événements menant à l'apoptose induite par le $\mathrm{TNF} \alpha$, Hannun [12] situe en amont l'activation de la cPLA2. L'AA libéré active la sphingomyélinase (figure 2). Le céramide produit conduit à l'activation des protéases de la famille des interleukin converting enzyme (ICE ou caspases) qui sont directement impliquées dans le processus apoptotique. Ces résultats désignent l'AA comme messager de l'apoptose. Le céramide, en aval, ne serait qu'un relais.

C'est seulement récemment que l'apoptose a été reconnue comme une composante essentielle de maladies cardiaques, dont l'insuffisance cardiaque, l'hypertrophie, les myocardites virales, les atteintes post-ischémiques. Le rôle de l'AA dans l'apoptose des cardiomyocytes n'a pas été formellement établi. Cependant, les quelques résultats concernant les mécanismes de l'apoptose cardiaque n'infirment pas cette hypothèse. Ainsi, l'étude pionnière qui montre que le TNF $\alpha$ induit l'apoptose des cardiomyocytes ventriculaires de rat adulte, implique la cascade des sphingolipides dans le processus apoptotique [36]. Les auteurs montrent que le céramide C2 induit l'apoptose des cardiomyocytes, mais ils insistent sur le rôle d'intermédiaire potentiel de la sphingosine, produit d'hydrolyse du céramide par la céramidase: après 10 minutes d'exposition au TNF $\alpha$, la concentration de sphingosine dans les cardiomyocytes est augmentée de $60 \%$. Ajoutée au milieu extracellulaire, à une concentration de $10 \mu \mathrm{M}$ pendant $18 \mathrm{~h}$, la sphingosine induit l'apoptose de $95 \%$ des cardiomyocytes. Dans les mêmes conditions et à la même concentration, le céramideC2 n'induit l'apoptose que de $32 \%$ des cellules. Cette étude n'a pas abordé la question de l'implication, possible, de la PLA2 et de l'AA en amont de la libération de sphingosine. On pourrait, en effet, supposer la cascade d'événements décrite pour les cellules HL-60: l'activation de la cPLA2 par le TNF $\alpha$ entraînerait l'activation de la cPLA2 et une libération d'AA qui provoquerait l'activation de la sphingomyélinase. Le céramide produit serait converti en sphingosine. S'agissant de la réponse apoptotique des cardiomyocytes à l'angiotensine II, les seules informations sur le mécanisme mis en jeu concernent la translocation des isoformes $\varepsilon$ et $\delta$ de la protéine-kinase $\mathrm{C}$ du cytosol vers la fraction membranaire et une élévation $\mathrm{du}\left[\mathrm{Ca}^{2+}\right]_{\mathrm{i}}$ qui serait consécutive à l'activation des protéine-kinases $\mathrm{C}$ [37]. Ces résultats n'éliminent pas l'hypothèse d'une implication de l'AA ou de l'un des dérivés sphingolipides: l'activation de la cPLA2 pourrait suivre l'augmentation de $\left[\mathrm{Ca}^{2+}\right]_{\mathrm{i}}$. La reperfusion, après un épisode ischémique, déclenche aussi le processus apoptotique dans les cardiomyocytes [38]. Plusieurs études ont décrit une accumulation d'AA dans les tissus ischémiques reperfusés (pour une revue voir [39]). A l'aide d'inhibiteurs de PLA2 il a été établi que la libération d'AA n'était pas un épiphénomène, lié à une atteinte de la membrane cellulaire, mais qu'elle se produisait au cours d'une phase réversible de l'épisode d'isché$\mathrm{mie} /$ reperfusion (voir [39]). La relation de cause à effet entre apoptose et accumulation d'AA a été ignorée par les auteurs. Mais Bielawska et al. du groupe de Hannun [40] viennent de montrer qu' in vitro, sur des cardiomyocytes de rat nouveau-né, comme in vivo, dans le ventricule après occlusion de l'artère coronaire, un épisode ischémique provoque une accumulation de céramide. Deux arguments conduisent les auteurs à considérer l'accumulation de céramide comme la cause de l'apoptose observée lors de la reperfusion: (1) l'analogue céramide C2 induit, in vitro, l'apoptose des cardiomyocytes de rat nouveau-né ; (2) la concentration de céramide revient à la normale dans les cellules qui survivent à la reperfusion, alors qu'elle reste élevée dans les cellules mortes ou mourantes. L'origine du céramide est discutée. Deux possibilités existent: la synthèse de novo de céramide ou l'hydrolyse de la sphingomyéline qui résulterait de l'activation de la sphingomyélinase. Dans un épisode ischémique, qui produit rapidement une déplétion en ATP et en créatine phosphate, la synthèse $d e$ novo est peu probable. Les auteurs favorisent donc l'hypothèse de l'hydrolyse de la sphingomyéline mais, là encore, sans faire référence à l'accumulation d'AA observée, par d'autres, au cours de l'ischémie.

Il apparaît donc que la libération d'AA et la cascade des sphingoli- pides, dans les cardiomyocytes, constituent une voie pour le déclenchement de l'apoptose. Ce n'est pas la seule. La réponse apoptotique des cardiomyocytes au facteur natriurétique (ANF) est relayée par le GMP cyclique [41]; l'apoptose déclenchée par l'étirement est liée à la formation de radicaux oxygène [42]. Il se peut que ces voies soient interdépendantes. Ainsi, la production de radicaux oxygène, qui survient en particulier lors de la reperfusion après un épisode ischémique, peut conduire à la peroxydation des lipides membranaires et à leur hydrolyse par la PLA2.

\section{Rôle messager de l'AA dans l'action cardiaque du mini-glucagon}

Nous avions montré que le métabolite (19-29) du glucagon, ou mini-glucagon, participait à l'action inotrope positive du glucagon sur la contraction cardiaque [43, 44]. Cependant, le mécanisme d'action restait à définir, le mini-glucagon n'ayant aucun effet sur les activités des adénylyl et guanylyl cyclases, phosphodiestérases ou phospholipase C, ni sur les concentrations cellulaires d'AMP ou GMP cycliques. Nous avons donc examiné les effets du peptide sur le relargage d'AA qui est observé sous l'action d'agents inotropes positifs tels que l'angiotensine II [24], la bradykinine [26], l'endothéline [25], les agonistes $\alpha$-adrénergiques [45]. En prenant pour modèle des cellules de ventricule cardiaque d'embryon de poulet chargées en AA $\left[{ }^{3} \mathrm{H}\right]$, nous montrons que le mini-glucagon, à concentrations subnanomolaires, augmente de façon dépendante de la dose la libération d'AA $\left[{ }^{3} \mathrm{H}\right]$ (figure 3). L'AA reproduit les effets du mini-glucagon lorsqu'il est ajouté au milieu extracellulaire à une concentration de $0,3 \mu \mathrm{M}$ : (1) dans les cardiomyocytes quiescents, l'AA augmente la charge en $\mathrm{Ca}^{2+} \mathrm{du}$ compartiment sarcoplasmique; (2) sur les cellules stimulées électriquement, l'AA potentialise les effets du glucagon, et ceux du 8-bromo-AMP cyclique, sur l'amplitude des transitoires calciques (voir légende de la figure 4) et sur l'amplitude de la contraction (figure 4). L'action de l'AA est directe (elle n'est pas blo- 
quée par les inhibiteurs des voies métaboliques de l'AA) et elle est spécifique [25]. Nous interprétons la potentialisation des effets du glucagon et de l'AMP cyclique par le miniglucagon, ou l'AA, comme étant la résultante: (1) de la capacité du mini-glucagon, via l'AA, d'induire une accumulation de $\mathrm{Ca}^{2+}$ dans le

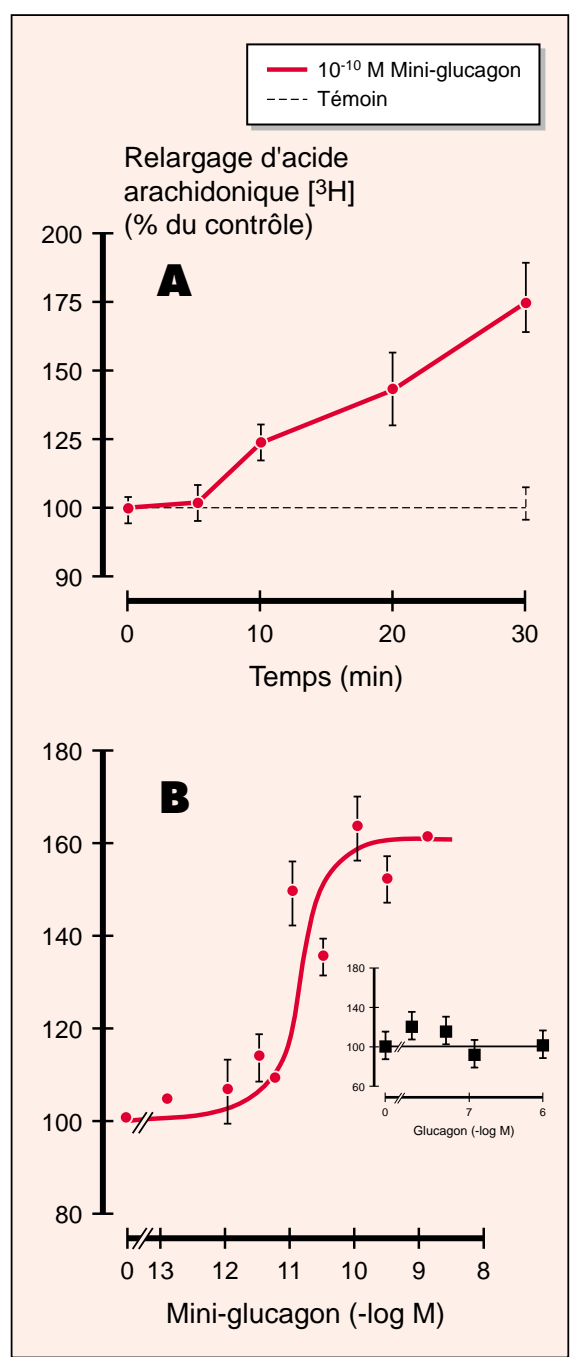

Figure 3. Le mini-glucagon augmente le relargage d'acide arachidonique $\left[{ }^{3} \mathrm{H}\right]$. Les cellules de ventricule de cour de poulet embryonnaire ont été marquées avec de l'acide arachidonique $\left[{ }^{3} \mathrm{H}\right]$. A. En présence de mini-glucagon $0,1 \mathrm{nM}$, le relargage $d^{\prime}$ acide arachidonique $\left[{ }^{3} \mathrm{H}\right]$ augmente jusqu'à $30 \mathrm{~min}$, alors qu'il reste constant dans les conditions basales. B. L'effet du mini-glucagon dépend de sa concentration [25]; le glucagon, à des concentrations supérieures, n'a pas d'effet sur le relar- compartiment du réticulum sarcoplasmique; (2) de la capacité du glucagon, via l'AMP cyclique et l'activation des canaux calciques de type L, de déclencher le relargage du $\mathrm{Ca}^{2+}$ de ce même compartiment. De fait, l'imagerie confocale montre que le relargage de $\mathrm{Ca}^{2+} \mathrm{du}$ compartiment $\mathrm{du}$ réticulum sarcoplasmique se déclenche spontanément et d'autant plus fréquemment que la charge en $\mathrm{Ca}^{2+} \mathrm{du}$ réticulum sarcoplasmique est importante [46]. L'AA est donc le second messager du mini-glucagon: il reste néanmoins à identifier sa cible. L'AA pourrait contrôler directement, ou indirectement, un système de transport de $\mathrm{Ca}^{2+}$ du réticulum sarcoplasmique: un canal $\mathrm{Ca}^{2+}$ ou la pompe à $\mathrm{Ca}^{2+} \mathrm{du}$ réticulum sarcoplasmique (SERCA).

\section{$A A$ et homéostasie calcique du cardiomyocyte}

Il n'y a pas de consensus pour les effets de l'AA sur l'homéostasie calcique et l'activité contractile du cardiomyocyte. Plusieurs études se sont appliquées à définir les effets de l'AA per se, grâce à des outils pharmacologiques, inhibiteurs des trois voies métaboliques principales: (1) les inhibiteurs des cyclo-oxygénases (ibuprofen, aspirine, indométhacine) ; (2) l'acide nordihydroguaiarétique (NDGA), inhibiteur des lipoxygénases; (3) le kétoconazole et le SKF 525A, inhibiteurs des cytochromes P450 .

Sur des cardiomyocytes de ventricule de rat adulte stimulés électrique-

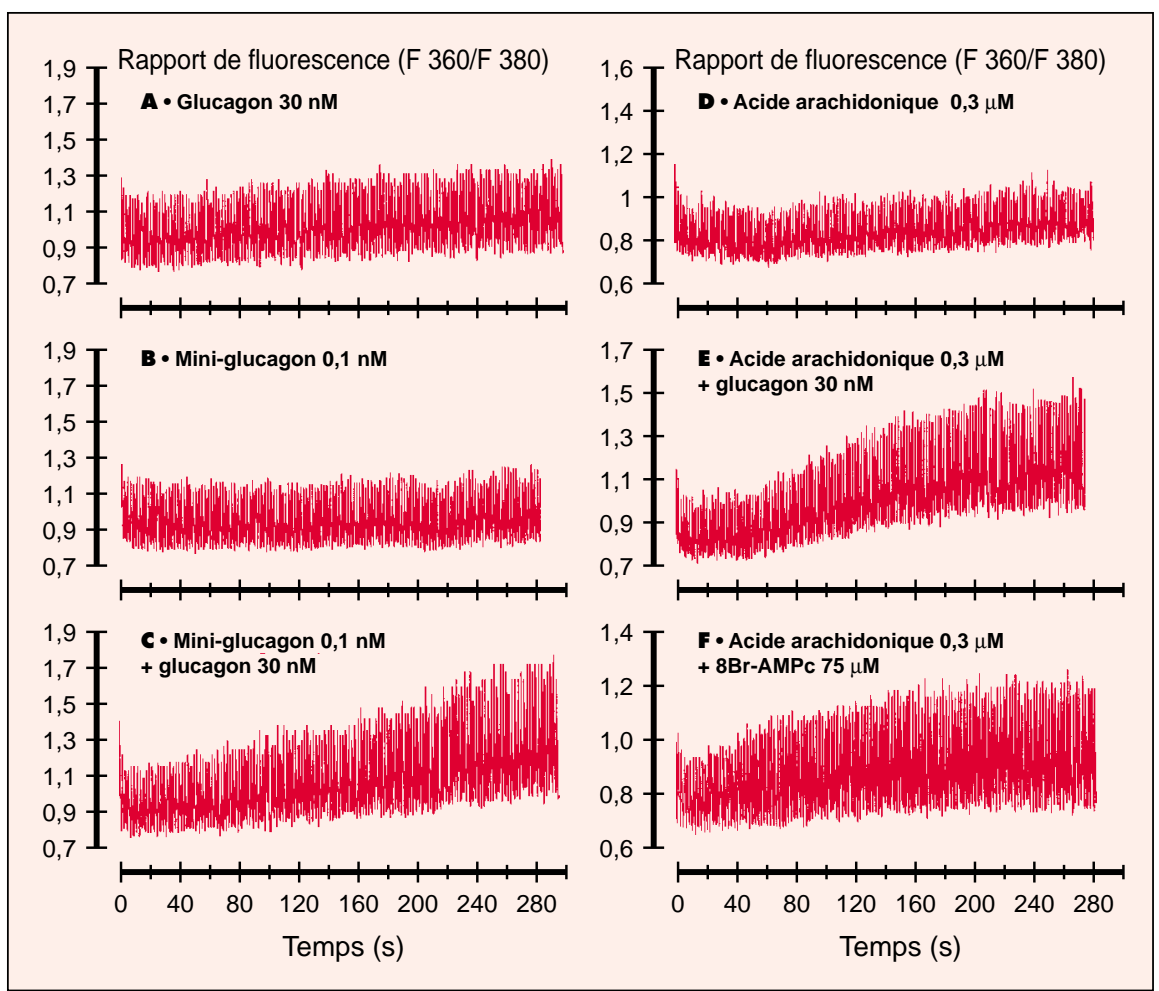

Figure 4. L'acide arachidonique reproduit l'effet du mini-glucagon sur les "transitoires calciques" des cellules stimulées électriquement. Les cellules de ventricule de cœur de poulet embryonnaire, chargées en Fura 2, sont stimulées électriquement à $0,5 \mathrm{~Hz}$. Chaque stimulation électrique déclenche une augmentation transitoire de la concentration de calcium libre cytosolique ou "transitoire calcique". Comme le mini-glucagon, l'acide arachidonique, ajouté à $0,3 \mu \mathrm{M}$ au milieu de perfusion des cellules, potentialise les effets du glucagon et de I'AMP cyclique sur l'amplitude des transitoires de $\mathrm{Ca}^{2+}$ cytosolique [25]. Les transitoires de $\mathrm{Ca}^{2+}$ cytosolique figurent la libération de $\mathrm{Ca}^{2+}$ du réticulum sarcoplasmique. Ils sont liés à l'activation des canaux calciques de type $L$, les ions $\mathrm{Ca}^{2+}$ entrant se liant aux canaux calciques du réticulum sarcoplasmique et déclenchant leur ouverture. Les pics de $\mathrm{Ca}^{2+}$ cytosolique déclenchent la contraction. 
ment, Damron et Summers (Cleveland, OH, USA) [47] montrent que l'AA, ajouté à la concentration de $50 \mu \mathrm{M}$ au milieu de perfusion, augmente et prolonge les transitoires calciques : cet effet est corrélé à l'activation de la protéine-kinase $\mathrm{C}$ et à l'inhibition des canaux $\mathrm{K}^{+}$dépendants du voltage. Les auteurs précisent que toutes les cellules ne répondent pas à l'AA et suggèrent qu'une hétérogénéité de réponse peut être attendue du fait de l'hétérogénéité de la distribution des canaux $\mathrm{K}^{+}$dans les zones ventriculaires. Il faut remarquer que dans une étude antérieure, Damron et al. [47], montraient un blocage par l'AA des transitoires calciques mais, dans cette première étude, l'AA était injecté en bolus dans le milieu cellulaire ce qui sousentend, dans les premiers instants de l'expérience, une concentration d'AA beaucoup plus élevée que la concentration finale indiquée.

Une étude [48], réalisée sur des cardiomyocytes de rat nouveau-né stimulés électriquement, montre que l'exposition des cellules à des concentrations de $10-30 \mu \mathrm{M}$ d'AA entraîne, après quelques minutes (4 à $9 \mathrm{~min}$ ), une diminution de la fréquence des oscillations calciques. Un arrêt complet des oscillations est observé après $15 \mathrm{~min}$. Ces effets propres de l'AA sont annulés si la différence de potentiel appliquée aux cellules est augmentée. Ces observations vont dans le sens de la diminution de l'excitabilité des cellules cardiaques par l'AA rapportée par Kang et al. (Boston, MA, USA) [49]. Elles doivent aussi être rapprochées des effets de l'AA qui ont été décrits sur les différents courants $\mathrm{Ca}^{2+}, \mathrm{Na}^{+}$et $\mathrm{K}^{+}$ [50]. Une diminution d'excitabilité peut être due, en effet, à une hyperpolarisation membranaire (le potentiel membranaire est déplacé vers les valeurs négatives) ou à une diminution de la vitesse de dépolarisation ou de repolarisation, et peut avoir pour origine des altérations des courants $\mathrm{Ca}^{2+}, \mathrm{Na}^{+}$ou $\mathrm{K}^{+}$. Hoffmann et al. (Cincinnati, OH, USA) [48] privilégient l'hypothèse d'effets de l'AA sur les systèmes de transport de $\mathrm{Ca}^{2+} \mathrm{du}$ sarcolemme et du réticulum sarcoplasmique.

En conclusion, ces études ne permettent pas d'établir un schéma linéaire du mécanisme d'action de l'AA. Mais toutes, exceptée la nôtre [25], ont utilisé des concentrations élevées d'AA (de 10 à $50 \mu \mathrm{M}$ ) qui se rapprochent des concentrations d'AA atteintes au cours de l'ischémie mais sont très supérieures aux concentrations d'AA libre, estimées dans le cardiomyocyte normal, auxquelles l'AA est susceptible de jouer un rôle de messager. On a cependant envie de retenir que les cibles potentielles de l'AA dans le cardiomyocyte sont: les canaux ioniques, la protéine-kinase $\mathrm{C}$ et la sphingomyélinase (figure 2).

\section{Conclusion}

L'AA joue un rôle de second messager. Ce rôle est particulier puisque l'AA relaie l'activation de récepteurs de type TNF- $\alpha$, en amont de l'activation en cascade de kinases, et celle de récepteurs couplés à des protéines $\mathrm{G}$ (mini-glucagon). Néanmoins, dans les deux exemples, le rôle de l'AA est différent. Dans le processus apoptotique déclenché par le TNF $\alpha$, l'AA transmet l'information (transmittal pathway) selon la terminologie de R. Iyengar (New York, USA) [51]. En revanche, dans la voie d'action du glucagon/mini-glucagon l'AA joue un rôle permissif pour la réponse à l'AMP cyclique (gating pathway). Cet effet «permissif» de l'AA, ou cette synergie AA/AMP cyclique n'est peut-être pas la moins intéressante et ne se limite peut-être pas au mécanisme d'action du glucagon/miniglucagon. On remarquera que l'activation simultanée des deux voies, AA et AMP cyclique, est associée à une efficacité particulière du glucagon en tant qu'agent inotrope positif [52]

\section{RÉFÉRENCES}

1. Bonventre JV, Huang Z, Taheri MR, O'Leary E, Li E, Moskowitz MA, Sapirstein A. Reduced fertility and postischaemic brain injury in mice deficient in cytosolic phospholipase A2. Nature 1997; 390: 622-5.

2. Uozumi N, Kume K, Nagase T, Nakatani N, Ishii S, Tashiro F, Komagata Y, Maki K, Ikuta K, Ouchi Y, Miyazaki J, Shimizu T. Role of cytosolic phospholipase A2 in allergic response and parturition. Nature 1997; 390 : 618-22.

3. Walsh DA, Van Patten SM. Multiple pathway signal transduction by the cyclic AMPdependent protein kinase. FASEB J 1994; 8 : 1227-36.

4. Muller B, Komas N, Keravis T, Lugnier C. Les phosphodiestérases des nucléotides cycliques. Med Sci 1993; 9: 1335-41.
5. Vaandrager AB, de Jonge HR. Signalling by cGMP-dependent protein kinases. Mol Cell Biochem 1996; 157: 23-30.

6. Ackerman MJ, Clapham DE. Ion Channels. Basic science and clinical disease. $N$ Engl J Med 1997; 336 : 1575-686.

7. Weiss FU, Daub H, Ullrich A. Novel mechanisms of RTK signa generation. Curr Opin Gen Dev 1997; 7 : 80-6.

8. Heldin $\mathrm{CH}$, Miyazono $\mathrm{K}$, ten Dijke P. TGF $\beta$ signalling from cell membrane to nucleus through SMAD proteins. Nature 1997; 390: 465-71.

9. Ihle JN. Cytokine receptor signalling. Nature 1995 ; 377 : 591-4.

10. Divecha N, Irvine RF. Phospholipid signaling. Cell 1995; 80 : 269-78.

11. Khan WA, Blobe GC, Hannun YA. Arachidonic acid and free fatty acids as second messengers and the role of protein kinase C. Cell Signal 1995; 7: 171-84.

12. Hannun YA. Functions of ceramide in coordinating cellular responses to stress. Science 1996 ; 274 : 1855-9.

13. Van Bilsen M, Van der Vusse GJ. Phospholipase-A2-dependent signalling in the heart. Cardiovasc Res 1995; 30 : 518-29.

14. Béréziat G. Diversité des phospholipases A2 et de leurs fonctions. C R Soc Biol 1996; 190 : 409-16.

15. Fourcade O, Simon MF, Leballe F, Gaigé B, Gaits F, Delagebeaudeuf C, Gassama A, Salles JP, Fauvel J, Chap H. Phospholipases A2 et pathologie inflammatoire: consensus et nouveaux concepts. Med Sci $1996 ; 12: 323-32$.

16. Clark JD, Schievella R, Nalefski EA, Lin LL. Cytosolic phospholipase A2. J Lipid Mediat Cell Signal 1995; 12: 83-117.

17. Leslie CC. Properties and regulation of cytosolic phospholipase A2. I Biol Chem 1997 ; 272: 16709-12.

18. Wolf MJ, Gross RW. The calcium-dependent association and functional coupling of calmodulin with myocardial phospholipase A2. J Biol Chem 1996; 271: 20989-92.

19. Wolf MJ, Wang J, Turk J, Gross RW. Depletion of intracellular calcium stores activates smooth muscle cell calcium-independent phospholipase A2. J Biol Chem 1997 ; 272: 1522-6.

20. Tang J, Kriz RW, Wolfman N, Shaffer M, Seehra J, Jones SS. A novel cytosolic calcium-independent phospholipase A2 contains eight ankyrin motifs. J Biol Chem $1997 ; 272$ : 8567-75.

21. Axelrod J. Phospholipase A2 and G proteins. Trends Neurosci 1995; 18: 64-5.

22. Lin LL, Lin AY, Knopf JL. Cytosolic phospholipase A2 is coupled to hormonally regulated release of arachidonic acid. Proc Natl Acad Sci USA 1992; 89: 6147-51.

23. Sadoshima J, Izumo S. Signal transduction pathways of angiotensin II-induced cfos gene expression in cardiac myocytes in vitro. Circ Res 1993; 73: 424-38. 


\section{RÉFÉRENCES}

24. Lokuta AJ, Cooper C, Gaa ST, Wang HE, Rogers TB. Angiotensin II stimulates the release of phospholipid-derived second messengers through multiple receptor subtypes in heart cells. J Biol Chem 1994; 269 : 4832-8.

25. Sauvadet A, Rohn T, Pecker F, Pavoine C. Arachidonic acid drives mini-glucagon action in cardiac cells. J Biol Chem 1997; 272: $12437-45$.

26. Nakamura F, Minshall RD, Le Breton GC, Rabito SF. Thromboxane A2 mediates the stimulation of inositol 1,4,5-trisphosphate production and intracellular calcium mobilization by bradykinin in neonatal rat ventricular cardiomyocytes. Hypertension 1996; 28 : 444-9.

27. Sadoshima J, Izumo S. Mechanical stretch rapidly activates multiple signal transduction pathways in cardiac myocytes: potential involvement of an autocrine/paracrine mechanism. EMBO J 1993; 12: 1681-92.

28. De Windt LJ, Willemsen PHM, Pöpping S, Van der Vusse GJ, Reneman RS, Van Bilsen M. Cloning and cellular distribution of a group II phospholipase A2 expressed in the heart. J Mol Cell Cardiol 1997; 29 : 2095-106.

29. McHowat J, Creer MH. Lysophosphatidylcholine accumulation in cardiomyocytes requires thrombin activation of $\mathrm{Ca}^{2+}$-independent PLA2. Am J Physiol 1997; 272: H1972-80.

30. McHowat J, Liu S. Interleukin-1beta stimulates phospholipase A2 activity in adult rat ventricular myocytes. Am J Physiol 1997; 272: C450-6.

31. Hazen SL, Ford DA, Gross RW. Activation of a membrane-associated phospholipase A2 during rabbit myocardial ischemia which is highly selective for plasmalogen substrate. J Biol Chem 1991; 266: 5629-33.

32. Hannun YA, Obeid LM. Ceramide: an intracellular signal for apoptosis. Trends Biochem Sci 1995; 20 : 73-7.

33. Jayadev S, Linardic CM, Hannun YA. Identification of arachidonic acid as a mediator of sphingomyelin hydrolysis in response to tumor necrosis factor alpha. J Biol Chem 1994; 269 : 5757-63.

34. Jayadev S, Hayter HL, Andrieu N, Gamard CJ, Liu B, Balu R, Havakawa M, Ito F, Hannun YA. Phospholipase A2 is necessary for tumor necrosis factor $\alpha$-induced ceramide generation in L929 cells. I Biol Chem 1997; 272 : 17196-203.

35. Hayakawa M, Ishida N, Takeuchi K, Shibamoto S, Hori T, Oku N, Ito F, Tsujimoto M. Arachidonic acid-selective cytosolic phospholoipase A2 is crucial in the cytotoxic action of tumor necrosis factor. J Biol Chem $1993 ; 268: 11290-5$

36. Krown KA, Page MT, Nguyen C, Zechner D, Gutierrez V, Comstock KL, Glembotski CC, Quintana PJE, Sabbadini RA. Tumor necrosis factor alpha-induced apoptosis in cardiac myocytes. J Clin Invest 1996; 98: 2854-65.

37. Kajstura J, Cigola E, Malhotra A, Li P, sin II induces apoptosis of adult ventricular myocytes in vitro. J Mol Cell Cardiol 1997; 29: 859-70.

38. Gottlieb RA, Burleson KO, Kloner RA, Babior BM, Engler RL. Reperfusion injury induces apoptosis in rabbit cardiomyocytes. J Clin Invest 1994; 94 : 1621-8.

39. Van der Vusse GJ, Reneman RS, Van Bilsen M. Accumulation of arachidonic acid in ischemic/reperfused cardiac tissue: possible causes and consequences. Prostaglandins Leukotr Essent Fatty Acids 1997; 57 : 85-93.

40. Bielawska AE, Shapiro JP, Jiang L, Melkonyan HS, Piot C, Wolfe CL, Tomei LD, Hannun YA, Umansky SR. Ceramide is involved in triggering of cardiomyocyte apoptosis induced by ischemia and reperfusion. Am J Pathol 1997; 151 : 1257-63.

41. Wu CF, Bishopric NH, Pratt RE. Atrial natriuretic peptide induces apoptosis in neonatal rat cardiac myocytes. J Biol Chem $1997 ; 272$ : 14860-6.

42. Cheng W, Li B, Kajstura J, Li P, Wolin MS, Sonnenblick EH, Hintze TH, Olivetti G, Anversa P. Stretch-induced programmed myocyte cell death. J Clin Invest 1995; 96 : 2247-59.

43. Pavoine C, Brechler V, Kervran A, Blache P, Le-Nguyen D, Laurent S, Bataille D, Pecker F. Mini-glucagon (glucagon [19$29]$ ) is a component of the positive inotropic effect of glucagon. Am J Physiol 1991; 260: C993-9.

44. Pecker F. Glucagon et mini-glucagon. Med Sci 1991; 7 : 911-7.

45. Kurachi $Y$, Ito $H$, Sugimoto $T$, Shimizu T, Miki I, Ui M. Alpha-adrenergic activation of the muscarinic $\mathrm{K}^{+}$channel is mediated by arachidonic acid metabolites. Pflugers Arch $1989 ; 414: 102-4$.

46. Cheng H, Lederer WJ, Cannell MB. Calcium sparks: elementary events underlying excitation-contraction coupling in heart muscle. Science 1993; 262: 740-4.

47. Damron DS, Summers BA. Arachidonic acid enhances contraction and intracellular $\mathrm{Ca}^{2+}$ transients in individual rat ventricular myocytes. Am J Physiol 1997; 272: H350-9.

48. Hoffmann P, Richards D, HeinrothHoffmann I, Mathias P, Wey H, Toraason M. Arachidonic acid disrupts calcium dynamics in neonatal rat cardiac myocytes. Cardiovasc Res 1995 ; 30 : 889-98.

49. Kang JX, Xiao YF, Leaf A. Free longchain polyunsaturated fatty acids reduce membrane electrical excitability in neonatal rat cardiac myocytes. Proc Natl Acad Sci USA 1995; 92: 3997-4001.

50. Bendahhou S, Cummins TR, Agnew WS. Mechanism of modulation of the voltagegated skeletal and cardiac muscle sodium channels by fatty acids. Am J Physiol 1997; 272: C592-600.

51. Iyengar R. Gating by cyclic AMP : expanded role for an old signaling pathway. Science 1996 ; 271 : 461-3.

52. Zaloga GP. Cardiovascular emergencies. In: Picazo J, ed. Glucagon in acute medicine. Pharmacological, clinical and therapeutic implications. Dordretch: Kluwer Academic Publishers, 1993: 11-25.

\section{Summary}

The messenger role of arachidonic acid in cardiomyocytes

Arachidonic acid (AA) is not only a major structural constituent of membranes and a key precursor of various bioactive molecules (eicosanoids in particular), but it is also a second intracellular messenger. Its release from membrane phospholipids occurs upon several hormonal stimuli and in the course of pathological situations, ischemia injury in particular. This mainly relies on the activation of phospholipases A2 (PLA2), but may also involve phospholipases $\mathrm{C}$ or $\mathrm{D}$, and results in an increase in the intracellular level of unesterified AA. The role of AA as a second messenger in cardiomyocytes is emerging. AA is a good candidate as the mediator of apoptosis triggered by $\mathrm{TNF} \alpha$, a role of $\mathrm{AA}$ which has been formally established in human leukemia cells. In this pathway, the cytosolic PLA2 (cPLA2) is the effector system and the sphingomyelinase which hydrolyzes sphingomyelin into ceramide is the target of AA. AA has also been identified as the second messenger of mini-glucagon, the Cterminal, 19-29 metabolite of glucagon: (1) mini-glucagon stimulates the cPLA2 enzyme and evokes a dose-dependent release of membranous arachidonyl; (2) AA, like mini-glucagon, acts synergistically with either glucagon or cyclic AMP to increase both the amplitude of $\mathrm{Ca}^{2+}$ transients and contraction of electrically stimulated cells. The target(s) of AA remain to be identified and possible candidates are PKC and ionic channels. In conclusion, AA may act as a second messenger of both TNFo-like receptors and $G$ protein linked receptors. Interestingly, as the messenger of $\mathrm{TNF} \alpha$ in apoptosis, AA acts as a «transmittal messenger ». In contrast, in the mediation of the positive inotropic effect of glucagon, which relies on both glucagon and mini-glucagon actions, AA acts as a "gating messenger » of cyclic AMP action. 